\title{
A percepção dos profissionais de saúde sobre o cuidado ao idoso com transtorno mental*
}

\author{
Maria Giovana Borges Saidel ${ }^{1}$ \\ (D) http://orcid.org/0000-0002-3259-1760 \\ Claudinei José Gomes Campos ${ }^{1}$ \\ (D) https://orcid.org/0000-0001-9587-6694
}

Objetivo: compreender as percepções da equipe de um Centro de Atenção Psicossocial sobre o cuidado ao idoso com transtorno mental. Método: estudo qualitativo com referencial teóricometodológico: Teoria das Representações Sociais. O campo de estudo foi um Centro de Atenção Psicossocial no interior do estado de São Paulo. Amostra composta por conveniência, fechada por exaustão, constituída por 12 profissionais. A coleta de dados foi realizada por meio da entrevista semiestruturada e estes foram analisados pela análise temática de conteúdo. Resultados: emergiram três categorias que evidenciam contradições sobre a percepção dos profissionais acerca do papel do CAPS. Essas contradições podem estar ancoradas nas representações sobre as dificuldades relatadas na assistência ao idoso, que se sobrepõem às dificuldades no cuidado ao indivíduo com transtorno mental. A ausência de estratégias para o atendimento cotidiano é abordada e a educação profissional é apontada como importante, porém ausente. $O$ desgaste do profissional em saúde mental emerge e a percepção sobre a falta de recursos humanos é revelada nos discursos. Essas problemáticas trazem uma representação de práticas insuficientes que refletirão no cuidado ao idoso com transtorno mental. Conclusão: o estudo contribui com esclarecimentos a serem abordados em estudos de intervenção para potencializar a transformação do cuidado.

Descritores: Recursos Humanos; Serviços de Saúde Mental; Assistência a Idosos; Transtornos Mentais; Psiquiatria Geriátrica; Serviços de Saúde Comunitária.

\section{Como citar este artigo}

Saidel MGB, Campos CJG. The perception of health professionals regarding the care for the elderly with mental disorders. SMAD, Rev Eletrônica Saúde Mental Álcool Drog. 2020;16(1):1-8. doi: https://dx.doi.org/10.11606/issn.1806-6976.smad.2020.153947 


\section{The perception of health professionals regarding the care for the elderly with mental disorders}

Objective: to understand the perceptions of the staff of a Psychosocial Care Center regarding the care for the elderly with mental disorders. Method: qualitative study with a theoretical and methodological framework: Theory of Social Representations. The field of study was a Psychosocial Care Center in the state of São Paulo. Convenience sampling was used, and the sample consisting of 12 professionals was determined by saturation. Data were collected by semi-structured interviews and analyzed by thematic content analysis. Results: three categories emerged, and they showed contradictions regarding the professionals' perceptions of the CAPS' role. Such contradictions may be supported on the representations concerning the difficulties reported in the care for the elderly, which overlap with those in the care for individuals with mental disorders. The lack of strategies for routine care is discussed, and professional education is mentioned as important although it is non-existent. The burnout of mental health professionals emerges, and the perception of the lack of human resources is revealed in the discourses. These problems bring about a representation of insufficient practices that will reflect on the care for the elderly with mental disorders. Conclusion: the study contributes with clarifications to be addressed in intervention studies in order to strengthen heath care change.

Descriptors: Manpower; Mental Health Services; Old Age Assistance; Mental Disorders; Geriatric Psychiatry; Community Health Services.

\section{La percepción de los profesionales de la salud sobre el cuidado al anciano con el trastorno mental}

Objetivo: comprender las percepciones del equipo de un Centro de Atención Psicosocial sobre el cuidado al anciano con trastorno mental. Método: estudio cualitativo con referencial teóricometodológico: Teoría de las Representaciones Sociales. El campo de estudio fue un Centro de Atención Psicosocial en el interior del estado de São Paulo. Una muestra compuesta por conveniencia, cerrada por agotamiento, constituida por 12 profesionales. La recolección de datos fue realizada, a través de la entrevista semiestructurada y el análisis de los datos del análisis temático de contenido. Resultados: surgieron tres categorías que evidencian contradicciones sobre la percepción de los profesionales acerca del papel del CAPS. Estas contradicciones pueden estar ancladas en las representaciones acerca de las dificultades relatadas en la asistencia al anciano que se superpone con dificultades en el cuidado al individuo con trastorno mental. La ausencia de estrategias para la atención cotidiana es abordada y la educación profesional es señalada como importante, pero ausente. El desgaste del profesional en salud mental emerge y la percepción sobre la falta de recursos humanos es revelada en los discursos. Estas problemáticas traen una representación de prácticas insuficientes que reflejará en el cuidado al anciano con trastorno mental. Conclusión: el estudio contribuye con aclaraciones para ser abordados en estudios de intervención para potenciar la transformación del cuidado.

Descriptores: Recursos Humanos; Servicios de Salud Mental; Asistencia a los Ancianos; Transtornos Mentales; Psiquiatría Geriátrica; Servicios de Salud Comunitaria. 


\section{Introdução}

O movimento da Reforma Psiquiátrica no mundo possibilitou uma transformação significativa do modelo de atenção em saúde mental. Ocorreu o redirecionamento da atenção para os serviços comunitários abertos em detrimento das práticas manicomiais e serviços de internação, além do desenvolvimento e aplicação dos conceitos relativos à prevenção em saúde mental. Essa realidade traz a premência iminente de instrumentalizar os profissionais e os serviços especializados que estão articulados com a assistência às pessoas com transtornos mentais $^{(1-2)}$.

Os recursos humanos para o cuidado em saúde mental nos países em desenvolvimento são motivo de preocupação evidenciada em estudos internacionais. Essa preocupação está relacionada tanto ao dimensionamento dos trabalhadores quanto à formação profissional para a realização do cuidado(3-4). Esse fato gera sobrecarga aos profissionais dessa especialidade(5-6).

Na tentativa de resolver tal problemática, esforços globais têm sido realizados com o objetivo de desenvolver estratégias para solucionar a falta generalizada da força de trabalho nesses países. Tais esforços resultaram na descrição de uma estrutura básica para auxiliar gestores de saúde mental no alcance de recursos humanos eficazes e sustentáveis. Nessa estrutura são evidenciados itens articulados e necessários para o desenvolvimento de recursos humanos: política, gestão da força de trabalho, finanças, educação, parcerias e liderança. Para contemplar esses temas nos serviços de saúde mental faz-se necessária uma abordagem interdisciplinar e sistêmica com a participação de diversos setores. Essa abordagem deve ocorrer por meio de instituições governamentais e não governamentais, instituições de pesquisa e formadoras de profissionais, equipes e usuários do sistema(7-9). A articulação dessas iniciativas seria o início de um trabalho transformador para alcançar o objetivo de ampliar e melhorar os cuidados aos indivíduos com transtornos mentais(10).

As dificuldades do cuidado em saúde mental ganham uma tônica quando ocorre com populações específicas e com muitas particularidades. Idosos com transtornos mentais de longo prazo vivenciam uma condição crônica, o que impõe aos profissionais relações longas nos serviços de saúde, permeadas de angústias e adversidades. Somam-se duas grandes especialidades desafiadoras na contemporaneidade: a saúde mental/ psiquiatria e a gerontologia(11). Portanto, estudos sobre os recursos humanos em saúde mental, particularmente para o cuidado ao idoso, são pertinentes devido à prioridade da temática nas agendas governamentais mundiais para essa população.

Frente aos impasses sobre os recursos humanos em saúde mental apontados pela literatura e pela equipe que oferece o cuidado ao idoso com transtorno mental, elaborou-se a seguinte questão de pesquisa: quais as representações sociais da equipe de um Centro de Atenção Psicossocial (CAPS) sobre o cuidado ao idoso com transtorno mental? Diante dessa questão de pesquisa, o presente estudo teve como objetivo compreender as percepções da equipe de um CAPS sobre o cuidado ao idoso com transtorno mental.

\section{Método}

Trata-se de um estudo qualitativo com referencial teórico-metodológico ancorado na Teoria das Representações Sociais (TRS). A TRS articula-se com a pesquisa partindo da construção dos processos de ancoragem e objetivação, bem como da construção dos significados atribuídos por meio do universo consensual e reificado(12). Assim, o referencial teórico-metodológico coloca em evidencia a relação constante entre a estrutura social e o sujeito e entre os sujeitos uns com os outros, resultando na edificação das representações sociais.

$O$ estudo foi realizado em um Centro de Atenção Psicossocial no interior do estado de São Paulo. A coleta de dados foi realizada por meio da entrevista semiestruturada com a seguinte questão norteadora: qual a sua percepção sobre o cuidado ao idoso com transtorno mental?

Os dados foram reunidos ao longo de um período de oito meses, novembro de 2013 a julho de 2014), realizando, assim, o engajamento prolongado(13) (período de aculturação). Os critérios de inclusão foram: profissionais com a matrícula profissional adscrita no campo de estudo e experiência mínima de um ano na rede de atenção psicossocial (RAPS, e os de exclusão: profissionais que, por qualquer motivo, estivessem afastados do serviço. A amostra foi composta por conveniência e fechada por exaustão, ou seja, todos os profissionais da equipe do CAPS foram entrevistados. As entrevistas foram realizadas em dias e horários diferentes, com espaço temporal de no mínimo três dias entre os encontros, o que permitiu a triangulação temporal(13) adequada. Foram entrevistados diversos profissionais de diferentes categorias e níveis de escolaridade, permitindo, assim, a triangulação pessoal. Preservando o anonimato dos sujeitos foram utilizadas as siglas NM para nível médio e NS para nível superior, seguidas do número da entrevista.

As entrevistas foram gravadas em áudio e transcritas integralmente. Utilizou-se a análise temática para analisar os dados. Trata-se de um método utilizado em diferentes estruturas teóricas com as seguintes etapas: familiarização com os dados, construção dos códigos iniciais, busca de temas, análise dos temas, definição e atribuição de temas e produção do relatório(14). 
O projeto do estudo foi submetido ao Comitê de Ética e Pesquisa da Universidade Estadual de Campinas (UNICAMP). Após aprovação iniciaram-se os processos de aculturação e posterior coleta de dados, com a anuência dos sujeitos por meio do Termo de Consentimento Livre e Esclarecido. O anonimato dos sujeitos foi garantido pela codificação alfanumérica, cuidando-se para que fossem omitidas transcrições que indicassem identidades. O número do parecer do Comitê foi 342.202.

\section{Resultados}

A amostra final foi composta por doze profissionais de saúde, sendo nove mulheres e três homens com a faixa etária entre 29 e 46 anos (dois enfermeiros, um médico, dois técnicos de enfermagem, um assistente social, dois terapeutas ocupacionais, um monitor, um auxiliar administrativo, um auxiliar de serviços gerais e um psicólogo). O tempo de atuação dos entrevistados na atenção psicossocial variou de 3 a 31 anos e, no campo de pesquisa (CAPS). foi de 3 a 8 anos.

A seguir são descritas as categorias temáticas que emergiram após a análise de dados para a compreensão das representações sociais da equipe.

Tema 1: Representação sobre o antagonismo do papel do Centro de Atenção Psicossocial na assistência ao idoso com transtorno mental

Os profissionais colocam a representação desse serviço ancorada em questões que causam apreensão e inquietude na equipe. Compreende-se que há preocupação nas falas em relação ao papel exercido na contemporaneidade pelo serviço quanto ao cuidado do idoso com transtorno mental. Me preocupo muito com a institucionalização do CAPS. Aqui é uma creche, substituto literalmente da internação, e o CAPS não é isso, não deveria ser isso (NS01); As pessoas usam o CAPS como creche, a família vem e deixa o idoso para passar o dia, muitas para poder trabalhar (NS02).

Existe a compreensão do serviço, porém a realidade apresenta-se contrária às diretrizes da política. Isso aqui não deveria ser uma creche, existe um período de chegada e um período de alta, ainda que essa alta não seja um desligamento total do serviço de saúde mental (NM03).

Além de questionamentos frente às condutas da equipe e ao trabalho individual do profissional. Eu não entendo algumas condutas aqui, alguns idosos são retirados de projetos porque o 'comportamento não condiz com o esperado', mas espera aí, qual é o nosso papel? Nós temos que ensinar o paciente a lidar com isso, mas vejo que minha voz é abafada (NM01).

Tema 2: Representação sobre a ausência de estratégias de desenvolvimento e aperfeiçoamento para a assistência ao idoso com transtorno mental
Evidenciou-se uma representação de que a equipe é insuficiente para o cuidado oferecido ao idoso com transtorno mental. Além da percepção de que essa população oferece uma demanda maior que outras. Eu acho que a equipe é muito pouco, eu acho que deveria ter muito mais gente, o trabalho é estressante, os idosos são mais difíceis, tem hora que eles estressam a gente" (NM04); Não temos gente suficiente para pensar nisso [práticas específicas aos idosos com transtornos mentais], é uma correria para tudo lado, todo dia, está bem difícil (NM02).

Além da percepção de falta de recursos humanos, a equipe também representa uma insatisfação pela ausência de educação interprofissional para a assistência a esses idosos. Não temos curso, não temos nada que nos ajude a assistir melhor esse idoso, fica difícil (NS04); Desde que estou aqui [três anos] nunca ouvi nem falar sobre isso, para atender idoso com transtorno, e a gente tem idosos aqui, mas nunca fiz curso, nada (NM04).

Na fala da equipe, há a representação sobre a responsabilidade da RAPS em ofertar essas capacitações. Não temos curso, nunca veio nada da Rede, e faz muita falta, pois a gente precisa se atualizar, e é a rede que tem dar né?" (NM03); Não temos capacitação para lidar com o idoso com transtorno mental, desde que eu estou aqui nunca tive nada sobre o assunto, acho que a secretaria [de saúde] deveria dar esses cursos, um apoio né? (NS07).

Tema 3: Representação sobre as fragilidades e potencialidade do trabalho em rede no cuidado ao idoso com transtorno mental

Há uma representação clara sobre a percepção da equipe acerca do trabalho em rede, além de esse entendimento estar ancorado em situações consideradas irrelevantes quando estas ocorrem em idosos na atenção básica. Falta bastante o trabalho em rede, porque muitas vezes, quando o paciente vem para o acolhimento ou para a triagem, a familia relata: 'Ah, mas ele é assim há tanto tempo, a gente achava que era normal' até entrar em surto. Faltou o PSF entende (NS05).

A ausência de procedimentos padronizados é apontada como uma possível causa de desencontros na rede. Existe um problema crônico nos serviços que não são especializados em transtornos mentais, é a ausência de protocolos. Não é só isso, lógico, mas já ajudaria" (NS06). A ausência de rede somada à deficiência de recursos também é apontada como um obstáculo para a prática das atividades. Uma rede adequada, primeiro, é aquela em que - seja o governo federal, municipal e estadual - nos dê um incentivo financeiro porque, hoje, sem dinheiro a gente não faz nada. Eu acho horroroso esse lugar, os pacientes não merecem estar aqui, a estrutura física... (NM02)

Entretanto, em meio às inúmeras adversidades dos processos de trabalho, os profissionais percebem e objetivam em parcerias, possibilidades de melhorias. $A$ gente está tentando desenvolver outras parcerias, a gente está 
começando a ter um contato um pouco melhor com a atenção básica, a Unidade de Saúde da Família, ainda com uma certa resistência, mas está um pouco melhor (NS01); Tem vezes que a gente precisa de ajuda, é CRAS [Centro de Referência de Assistência Social], é PSF, qualquer coisa já é uma ajuda. O problema é que a pessoa despeja aqui e não quer nem saber mais, aí fica complicado (NMO3)

\section{Discussão}

$\mathrm{Na}$ apresentação dos resultados da primeira categoria são evidenciadas contradições sobre o papel do CAPS no cuidado ao idoso com transtorno mental. Essas representações geram angústias, que, muitas vezes, podem fazer emergir uma sensação de perda da identidade profissional. Tal constatação gera outra questão: se o CAPS, na representação dos trabalhadores, perde o seu papel estratégico de reabilitação, então, qual é o papel dele nesse contexto?

Essas representações sociais foram construídas a partir de instrumentos internos e externos vivenciados por esses profissionais. Tais construções impactam as práticas cotidianas do serviço, pois os indivíduos atuam mediante suas representações advindas do universo consensual(12). Portanto, quando o CAPS é objetivado como uma creche, logo o idoso é uma criança e poderá ser assistido como tal.

O CAPS é um serviço aberto, especializado, comunitário e substitutivo à internação de pessoas com transtornos mentais. É considerado um dispositivo estratégico no movimento da Reforma Psiquiátrica, devido ao potencial de mudança e às ações de cuidado junto às pessoas em sofrimento psíquico(1). O trabalho dos profissionais do CAPS deverá estar articulado com a proposta do serviço descrito na política. É preciso criar espaços reflexivos que permitam a construção de ações efetivas durante esse processo de trabalho(2-4). Tais ações deverão estar pautadas nas seguintes diretrizes: reabilitação do indivíduo e geração de autonomia; diminuição da necessidade de internação hospitalar, reconstrução de identidades por meio das relações terapêuticas e promoção da saúde mental dos usuários ${ }^{(15-16)}$.

Retomando a representação do CAPS como uma creche e, consequentemente, o idoso como uma criança, pode-se inferir que há uma dificuldade em olhar e cuidar do idoso com transtorno mental. O idoso em sofrimento psíquico já é descrito na literatura como objeto de estigma múltiplo, pois somado ao sofrimento psíquico, ele é 'velho'. Essa visão pode constituir barreiras na relação de cuidado(11). Quando há a objetivação com representação de creche/criança ocorre a formação do núcleo figurativo e um processo de naturalização inconsciente dessa equipe ${ }^{(12)}$, limitando, dessa forma, os sentimentos de angústia(7). Contudo, é necessário superar essa representação para que os trabalhadores possam exercer o seu papel sem elementos figurativos que dificultam a construção dos processos efetivos do cuidado.

A realidade descrita na primeira categoria está articulada às representações que originaram a segunda categoria. A equipe representa que a dificuldade em oferecer o cuidado ao idoso com transtorno mental está ancorada na ausência de estratégias que possam auxiliar no aperfeiçoamento da prática profissional, além da escassez de recursos humanos.

Os processos de trabalho exercidos pela equipe interprofissional nos serviços de saúde mental especializados apresentam avanços na construção de um modelo de atenção psicossocial contemporâneo. Porém, os profissionais que atuam em ambiente psiquiátrico estão mais expostos a desgastes físicos e psíquicos ${ }^{(16)}$ e, portanto, demonstram contradições e inúmeras problemáticas inerentes à implantação de equipamentos de saúde, das quais destaca-se exatamente o que emergiu dos resultados desse estudo: escassez de recursos humanos e ausência de educação profissional. Essas questões repercutem na configuração dos processos de trabalho, nas relações dos atores e na dinâmica de assistência dos trabalhadores ${ }^{(16-19)}$.

O fato de essas representações sociais emergirem nas entrevistas demonstra um processo cognitivo que apresenta representações emancipadas. O grupo constrói, por meio de suas falas (linguagem), explicações inconscientes que justificam alguns obstáculos relacionados ao cuidado do idoso com transtorno mental(11).

Esse movimento de formação das representações sociais pode ser olhado de maneira positiva, mostrando que há soluções para as problemáticas e que elas estão nos recursos humanos existentes. Enquanto no primeiro tema sugere-se a constituição de espaços reflexivos, no segundo, parece ser mais apropriada a criação de parcerias com instituições de pesquisa e formadoras.

Essas parcerias, articuladas às necessidades da equipe de saúde mental, poderiam resultar em processos educativos acurados. Os processos educativos por si só não constituem a transformação da prática, contudo, podem trazer componentes importantes para tal.

Na última categoria temática dos resultados ocorre a representação das fragilidades e potencialidades da RAPS. A equipe responsabiliza dispositivos de saúde que compõem a rede por falhas no acolhimento inicial do idoso com transtorno mental. Apontam e percebem ausência de procedimentos que auxiliem nessa classificação e objetivam a necessidade de protocolos. Nesse sentido a representação do grupo sobre a ausência de protocolos sugere uma compreensão por meio do universo reificado das representações sociais. Esse entendimento pode ser objetivado como uma possível estratégia pensada pelos profissionais para sanar algumas problemáticas sobre a ausência do trabalho em rede. Esse movimento dialético 
proporcionado pelas entrevistas é substancial para as mudanças de paradigma.

Estudos sobre processos de trabalho e avaliação dos CAPS evidenciam que as instituições e os profissionais deverão estar articulados para que a reorientação do modelo de atenção em saúde mental seja efetiva. O trabalho em rede é uma necessidade, e uma estratégia abordada pelo estudo é a condução simultânea dos projetos terapêuticos pelo CAPS e demais serviços de saúde. Essa ação seria um indicador que objetiva compreender o trabalho interprofissional e intersetorial(20-21).

Diversas inovações estão em curso em todo o mundo para medir e melhorar a qualidade dos cuidados em saúde mental. Essas iniciativas combinam os avanços na tecnologia do cuidado e oferecerem a possiblidade de construção de indicadores passíveis de serem avaliados. O objetivo é um cuidado em saúde mental em constante processo de avaliação e melhorias. Sendo assim, mais uma estratégia seria a partir das representações da equipe sobre a ausência de rede e protocolos, a construção de indicadores para avaliação dessas representações ${ }^{(1,10,20)}$.

Com os resultados poderiam conceber estratégias mais acuradas em pontos específicos da rede para aumentar a eficiência e um exemplo seriam a estratégia de referência, capacitação para aplicação de escalas e construção de protocolos de acolhimento e atendimento(20-21).

A eficiência do trabalho na RAPS é fundamental para a qualidade de assistência aos idosos com transtornos mentais. Muitas comorbidades podem causar imprecisão no acolhimento inicial na atenção básica e ser atribuídas apenas como particularidades do processo de envelhecimento. Essa confusão causaria, então, negligência e atrasos no tratamento dos transtornos mentais nos serviços especializados ou mesmo em outros dispositivos da rede, dependendo da repercussão e do sofrimento psíquico na vida desse idoso.

Profissionais qualificados em gestão e suporte se saúde são vitais para supervisionar a implementação de estratégias para alcançar melhores níveis de atenção em saúde mental( ${ }^{(6,10)}$. É preciso investimento para aumentar a capacidade de gestão nas redes de atenção psicossocial. Além disso, é necessário produzir protocolos e realizar o treinamento necessário para sua execução na prática cotidiana(17,21).

Os processos de avaliação também constituem ação importante. Os profissionais e os serviços de saúde necessitam de uma lista de indicadores com resultados validados, centrados no indivíduo usuário do serviço, bem como para populações especiais ${ }^{(10)}$, como por exemplo, os idosos. Faz-se necessária uma base de evidências para medidas de qualidade por meio de diretrizes práticas e monitoramento contínuo realizado por grupos pré-definidos que fornecerão estratégias para incorporar a melhoria da qualidade onde necessário e reduzir a sobrecarga percebida pelos profissionais ${ }^{(5,10,21)}$.

O presente estudo limita-se à compreensão das representações sociais da equipe sobre a assistência ao idoso com transtorno mental. Sendo assim, essa discussão pode contribuir para uma generalização naturalística em contextos similares. A literatura internacional mostra-se relevante, porém em contextos que já suplantaram os desafios representados pelos profissionais do presente estudo. Todavia, oferece diretrizes para assistências qualificadas com o uso de instrumentos que aperfeiçoam a assistência em saúde mental.

\section{Conclusão}

Os achados deste estudo evidenciaram que a assistência ao idoso com transtorno mental no serviço especializado representada pela equipe interprofissional é complexa e enfrenta inúmeras adversidades. Essa complexidade é diluída em três grandes proposições: os desafios sobre a identidade do serviço especializado comunitário em saúde mental na contemporaneidade, a ausência de competências e habilidades dos profissionais para a assistência a essa população específica e a insuficiência da RAPS para a condução adequada desses casos

Fazem-se necessários o enfrentamento e superação dos obstáculos que se apresentam no processo de assistência a essa população. As equipes devem incorporar momentos dialéticos nos serviços com orientações práticas e viáveis sobre a prática cotidiana dessa assistência peculiar, que mostra tendências de crescimento, além de parcerias, construção de instrumentos para avaliação e indicadores e investimentos na RAPS. É preciso aprimorar a construção de saberes sobre essa temática sem reduzir a conceitos, ampliando, dessa forma, a práxis e incorporando as subjetividades dos sujeitos. Essa prática é desafiadora, mas fundamental para alcançar a ampliação do entendimento e da prática da equipe para uma assistência integral e efetiva aos idosos com transtornos mentais.

A compreensão dessas mudanças nos contextos de prática, o acompanhamento adequado nos dispositivos de saúde e a implementação das estratégias mencionadas nesse artigo podem possibilitar movimentos fundamentais para uma futura transformação do cuidado ao idoso em saúde mental.

\section{Referências}

1. Tavares CMM, Muniz MP, Silva T. Priorities for research on mental health and the transformation of care model. 
RPESM. [Internet]. 2015; (esp2):107-12 [cited Sep 3 2018]. Avaliable from: http://www.scielo.mec.pt/ scielo.php?script $=$ sci_arttext\&pid $=$ S1647-21602015 000100018\&lng=pt\&nrm=iso\&tlng=pt.

2. Saias T, Laurentine VCD, Xavier B. Prevention in mental health: Social representations from French professionals. Health Sociol Rev. [Internet]. 2014;23(2):159-64 [cited Sep 3 2018]. doi: https://doi.org/10.1080/ 14461242.2014.11081970

3. Alves SR, Santos RP, Oliveira RG, Yamaguchi MU. Mental health services: perception of nursing in relation to overload and working conditions. J Res Fundam Care Online. [Internet] 2018;10(1): 25-29. doi: http://dx.doi.org/ 10.9789/2175-5361.2018.v10i1.25-29.

4. Marcolino TQ, Fantinatti EM, Gozzi APNF. Community of practice and mental health care: a systematic revision. Trab Educ Saúde. [Internet] 2018; 16(2):643-658 [cited Oct 4 2018] .doi: http://dx.doi. org/10.1590/1981-7746-sol00112.

5. Souza IAS, Pereira MO, Oliveira MAF, Pinho PH, Gonçalves RMDA. Work process and its impact on mental health nursing professionals. Acta Paul Enferm. [Internet] 2015;28(5):447-453 [cited Oct 5 2018]. doi: http://dx.doi.org/10.1590/1982-0194201500075.

6. Kakuma R, Minas H, Van Ginneken N, Dal Poz MR, Desiraju K, Morris JE, Saxena S, Scheffler RM. Human resources for mental health care: current situation and strategies for action. Lancet. [Internet] 2011; 378(9803):1654-63. [cited Oct 14 2018]. doi: https:// doi.org/10.1016/S0140-6736(11)61093-3.

7. Bee P, Brooks H, Fraser C, Lovell K. Professional perspectives on service user and carer involvement in mental health care planning: a qualitative study. Int J Nurs Stud. [Internet] 2015; 52(12):1834-45. [cited Oct 14 2018]. doi: https://doi.org/10.1016/ j.ijnurstu.2015.07.008.

8. Ivbijaro G, Patel V, Chisholm D, Goldberg D, Khoja TA, Edwards TM, et al. Informing mental health policies and services in the EMR: costeffective deployment of human resources to deliver integrated community-based care. East Mediterr Health J. [Internet] 2015; [Acesso Oct 14 2018] 21(7):486-92. Avaliable from: http://www.who.int/iris/ handle/10665/255241

9. Van Ginneken N, Maheedhariah MS, Ghani S, Ramakrishna J, Raja A, Patel V. Human resources and models of mental healthcare integration into primary and community care in India: Case studies of 72 programmes. PLoS One. [Internet] 2017;12(6) [cited 14 Oct 2018]. doi: https://doi.org/10.1371/journal. pone. 0178954 .

10. Kilbourne AM, Beck K, Spaeth-Rublee B, Ramanuj P, O'Brien R, Tomoyasu N, Pincus $\mathrm{H}$. Measuring and improving the quality of mental health care: a global perspective. WId Psychiatry. [Internet] 2018;17(1):30-8 [cited Oct 15 2018]. doi: https://doi.org/10.1002/wps.20482.

11. Medeiros B, Foster J. Mental ill health in the elderly: medical students' social representations in the United Kingdom. Rev Esc Enferm USP. [Internet] 2014; 48(esp2):132-8. [cited Nov 02 2018]. doi: http://dx.doi. org/10.1590/S0080-623420140000800020

12. Moscovici S. Representações sociais: investigações em psicologia social. $8^{a}$ ed. Rio de Janeiro/Petrópolis: Vozes; 2011.

13. Braun V, Clarke V. Using thematic analysis in psychology. Qual Res Psychol. [Internet] 2006;3(2):77-101 [cited Nov 5 2018]. doi: https://doi. org/10.1191/1478088706qp063oa

14. Pedrosa TB, Moreira MIB. Mental health and justice: network care challenges. Rev Bras Pesq Saúde. [Internet] 2017;19(2):73-82 [cited Nov 05 2018]. doi: https://doi.org/10.21722/rbps.v19i2.18864.

15. Brolese DF, Lessa G, Santos JLG, Mendes JS, Cunha KS, Rodrigues $\mathrm{J}$. Resilience of the health team in caring for people with mental disorders in a psychiatric hospital. Rev Esc Enferm USP. [Internt] 2017; 51: e03230 [cited Nov 6 2018]. doi: http://dx.doi.org/10.1590/ s1980-220x2016026003230.

16. Clementino FS, Miranda FAN, Martiniano CS, et al. Satisfaction and work overload evaluation of employees' of Psychosocial Care Centers. Rev Fund Care Online. [Internet] 2018; 10(1):153-9 [cited Nov 06 2018]. doi: http://dx.doi.org/10.9789/2175-5361.2018. v10i1.153-159.

17. Pinho ES, Souza ACS, Esperidião E. Working processes of professionals at Psychosocial Care Centers (CAPS): an integrative review. Ciênc Saúde Coletiva. [Internet] 2018; 23(1):141-52. [cited Nov 6 2018]. doi: http:// dx.doi.org/10.1590/1413-81232018231.08332015.

18. Ohlsson R. Diagnosis as a Resource in the Social Representation of Mental Illness. Papers Soc Representations. [Internet] 2016;25(1):153-59 [cited Nov 6 2018]. doi: http://dx.doi.org/10.9789/21755361.2018.v10i1.153-159.

19. Onocko-Campos R, Furtado JPereira, Trapé TL, Emerich BF, Surjus LTLS. Evaluation Indicators for the Psychosocial Care Centers Type III: results of a participatory design. Saúde Debate. [Internet] 2017;41(spe):71-83 [cited Nov 6 2018]. doi: http:// dx.doi.org/10.1590/0103-11042017s07.

20. Twomey C, Prina AM, Baldwin DS, Das-Munshi J, Kingdon $D$, Koeser $L$, et al. Utility of the Health of the Nation Outcome Scales (HoNOS) in Predicting Mental Health Service Costs for Patients with Common Mental Health Problems: Historical Cohort Study. PLoS One. [Internet] 2016; 11(11):e0167103 [cited Nov 6 
2018]. doi: https://doi.org/10.1371/journal.pone.

0167103.

\section{Contribuição dos autores}

Concepção e desenho da pesquisa: Maria Giovana Borges Saidel, Claudinei José Gomes Campos. Obtenção de dados: Maria Giovana Borges Saidel, Claudinei José Gomes Campos. Análise e interpretação dos dados: Maria Giovana Borges Saidel, Claudinei José Gomes Campos. Análise estatística: Maria Giovana Borges Saidel, Claudinei José Gomes Campos. Obtenção de financiamento: Maria Giovana Borges Saidel, Claudinei José Gomes Campos. Redação do manuscrito: Maria Giovana Borges Saidel, Claudinei José Gomes Campos. Revisão crítica do manuscrito quanto ao conteúdo intelectual importante: Maria Giovana Borges Saidel.

Todos os autores aprovaram a versão final do texto.

Conflito de interesse: os autores declararam que não há conflito de interesse.

Esta licença permite que outros remixem, adaptem e criem a partir do seu trabalho para fins não comerciais, e embora os novos trabalhos tenham de lhe atribuir o devido crédito e não possam ser usados para fins comerciais, os usuários não têm de licenciar esses trabalhos derivados sob os mesmos termos. 